\title{
Optimizing methods for the study of intravascular lipid metabolism in zebrafish
}

\author{
KAN CHEN, CHANG-QIAN WANG, YU-QI FAN, YU-SHUI XIE, ZHAO-FANG YIN, ZUO-JUN XU, \\ HUI-LI ZHANG, JIA-TIAN CAO, ZHI-HUA HAN, YUE WANG and DONG-QIANG SONG
}

\author{
Department of Cardiology, Shanghai Ninth People's Hospital Affiliated to Shanghai Jiaotong University \\ School of Medicine, Shanghai 200011, P.R. China
}

Received December 30, 2013; Accepted September 18, 2014

DOI: $10.3892 / \mathrm{mmr} .2014 .2895$

\begin{abstract}
The zebrafish (Danio rerio) is a useful vertebrate model for use in cardiovascular drug discovery. The present study aimed to construct optimized methods for the study of intravascular lipid metabolism of zebrafish. The lipophilic dye, Oil Red O, was used to stain fasting zebrafish one to eight days post-fertilization (dpf) and to stain 7-dpf zebrafish incubated in a breeding system containing $0.1 \%$ egg yolk as a high-fat diet (HFD) for $48 \mathrm{~h}$. Three-dpf zebrafish were kept in CholEsteryl boron-dipyrromethene (BODIPY) 542/563 C11 water for $24 \mathrm{~h}$ which indicated the efficiency of CholEsteryl BODIPY 542/563 C11 intravascular cholesterol staining. Subsequently, 7-dpf zebrafish were incubated in water containing the fluorescent probe CholEsteryl BODIPY 542/563 C11 and fed a high-cholesterol diet (HCD) for $10 \mathrm{~d}$. Two groups of 7-dpf zebrafish were incubated in regular breeding water and fed with a regular or HCD containing CholEsteryl BODIPY 542/563 C11 for $10 \mathrm{~d}$. Finally, blood lipids of adult zebrafish fed with regular or HFD for seven weeks were measured. Oil Red $\mathrm{O}$ was not detected in the blood vessels of 7-8-dpf zebrafish. Increased intravascular lipid levels were detected in 7-dpf zebrafish incubated in $0.1 \%$ egg yolk, indicated by Oil Red $\mathrm{O}$ staining. Intravascular cholesterol was efficiently stained in 3-dpf zebrafish incubated in breeding water containing CholEsteryl BODIPY 542/563 C11; however, this method was inappropriate for the calculation of intravascular fluorescence intensity in zebrafish $>7$-dpf. In spite of this, intra-aortic fluorescence intensity of zebrafish fed a HCD containing CholEsteryl BODIPY 542/563 C11 was significantly higher $(\mathrm{P}<0.05)$ than that of those fed a regular diet containing CholEsteryl BODIPY 542/563 C11. The serum
\end{abstract}

Correspondence to: Dr Chang-Qian Wang or Dr Yu-Qi Fan, Department of Cardiology, Shanghai Ninth People's Hospital Affiliated to Shanghai Jiaotong University School of Medicine, 639 Zhi Zaoju Road, Shanghai 200011, P.R. China

E-mail:wangchangqian3@gmail.com

E-mail: fanyuqi773@gmail.com

Key words: Oil Red O, intravascular lipids, fluorescent probe, high throughput total cholesterol and triglyceride levels of adult zebrafish fed a HFD were markedly increased compared to those of the control group $(\mathrm{P}<0.05)$. In conclusion, the use of Oil Red $\mathrm{O}$ staining and CholEsteryl BODIPY 542/563 C11 may have applications in zebrafish intravascular lipid metabolism research and screens for novel lipid-regulating drugs.

\section{Introduction}

Abnormal lipid metabolism is associated with numerous diseases, including hepatic adipose infiltration, pancreatitis, obesity and diabetes (1-3). In addition, abnormal intravascular lipid metabolism is a major promoter of atherosclerosis, which may deteriorate into coronary disease and cerebral arterial thrombosis. Zebrafish (Danio rerio), with transparent embryos, external fertilization, clutch size and moderate generation time has functioned as an important vertebrate model $(4,5)$. There have been numerous lipid studies focused on the alimentary system, saccus vitellinus, angiogenesis and the nervous system $(6,7)$. Conversely, the study of zebrafish intravascular lipid metabolism has been limited, although studies have proven that zebrafish have similar digestive physiology and lipid metabolism to that of humans and the treatment of zebrafish with antihyperlipidemic drugs triggers similar responses to those observed in humans (8-11). Therefore, the establishment of a standardized platform for antihyperlipidemic drug screening in a zebrafish system is important.

Lipophilic dyes, including Oil Red O, Sudan Black B and Nile red, are tools used for the visualization of lipids in cells (12). The intravascular lipids of embryonic zebrafish are dyed by Oil Red O. Schlegel and Stainier (13) reported that the vasculature of zebrafish no longer had stainable neutral lipids six days post fertilization (dpf). Therefore, the changes in intravascular lipids among 1-8-dpf zebrafish were observed using Oil Red O staining in the present study.

There are numerous varieties of fluorescent sterol analogs commercially available; a novel boron-dipyrromethene (BODIPY)-tagged cholesterol analog was created with a modified fluorophore linker and this analog was able to be incorporated into cholesterol-rich regions of cells (14). Stoletov et al (11) discovered the deposition of red fluorescent cholesterol in vessel walls following feeding 5-dpf zebrafish a diet containing a fluorescent probe (CholEsteryl BODIPY 
576/589-C11) for ten consecutive days. This method was used in order to study intravascular cholesterol metabolism. As CholEsteryl BODIPY 576/589-C11 has been out of production since May 2012, it was necessary to identify a novel fluorescent probe and develop an analogous experimental protocol.

In the present study, the intravascular lipid metabolism of embryonic zebrafish was investigated using Oil Red $\mathrm{O}$ and CholEsteryl BODIPY 542/563 C11. Furthermore, the effects of a high-fat diet (HFD) on adult zebrafish intravascular lipid metabolism were studied. It was hypothesized that the optimized protocol developed in the present study may be used to investigate the gene functions associated with zebrafish lipid metabolism and to screen novel lipid-lowering drugs.

\section{Materials and methods}

Zebrafish. The zebrafish used in the present study were obtained from the Shanghai Research Center for Model Organisms (Shanghai, China), and the facilities for housing these animals were accredited by the Association for Assessment and Accreditation of Laboratory Animal Care International (Frederick, MD, USA). Zebrafish [AB strain and fli1:enhanced green fluorescent protein (EGFP) strain] were raised and maintained in $\mathrm{pH} 7.0$ reverse-osmosis-purified water under a 14 -h light $/ 10$-h dark photoperiod at $28^{\circ} \mathrm{C}$. Embryos were raised in $15-\mathrm{cm}$ Petri dishes following fertilization and adolescent fish were housed in 21 tanks with $~ 10$ fish per tank. A total of $307-d p f$ fasting wild-type zebrafish were incubated in breeding water containing $0.1 \%$ egg yolk for $48 \mathrm{~h}$. Wild-type zebrafish ( $3 \mathrm{dpf}, \mathrm{n}=30$ ) were incubated in breeding water containing $0.1 \mathrm{ppm}$ CholEsteryl BODIPY 542/563 C11 (Invitrogen Life Technologies, Carlsbad, CA, USA) for $24 \mathrm{~h}$. One group of 30 7-dpf zebrafish were incubated in water containing the fluorescent probe CholEsteryl BODIPY 542/563 C11 and fed a high-cholesterol diet (HCD) for $10 \mathrm{~d}$. Another two groups of 7-dpf zebrafish ( $\mathrm{n}=30$ /group) were incubated in regular breeding water and fed with a regular, or HCD containing CholEsteryl BODIPY 542/563 C11 for $10 \mathrm{~d}$. A total of 100 male zebrafish (age, 13 weeks) were randomly divided into two groups and fed a regular diet thrice daily (control group), or a HCD twice daily and a high-fat egg-yolk diet once daily (HCD group) for seven weeks. Animal experiments were approved by the Institutional Animal Care and Use Committee of Shanghai Research Center for Model Organisms (SRCMO) (Shanghai, China) and performed in accordance with their ethical standards. The distribution and regeneration of blood vessels were observed using fli1:EGFP zebrafish, in which blood vessel endothelial cells express EGFP.

Drug preparations. A stock solution of CholEsteryl BODIPY 542/563 C11 was produced by dissolving $1 \mathrm{mg}$ CholEsteryl BODIPY 542/563 C11 in $2 \mathrm{ml}$ chloroform (Sinopharm Chemical Reagent Co., Ltd., Shanghai, China) (stored at $-20^{\circ} \mathrm{C}$ ). Subsequently, $20 \mu 1$ stock solution was transferred into Eppendorf tubes (EP). Once the chloroform was completely evaporated, $10 \mathrm{ppm}$ solution was made by dissolving the powder into dimethyl sulfoxide (DMSO; Sangon Biotech Co.,Ltd., Shanghai, China). The final concentrations of CholEsteryl BODIPY 542/563 C11 and DMSO in breeding water were $0.1 \mathrm{ppm}$ and $0.1 \%(\mathrm{v} / \mathrm{v})$, respectively.
A working solution of the narcotic drug 3-aminobenzoic acid ethylester methanesulfate (MS-222; 200 ppm) was prepared by dissolving $20 \mathrm{mg}$ MS-222 (Fluka, SigmaAldrich, St. Louis, MO, USA) in $100 \mathrm{ml}$ double distilled water (SRCMO).

A $0.5 \%(w / v)$ stock solution of Oil Red O was prepared by adding solid Oil Red O (Sigma-Aldrich) to isopropanol (Sinopharm Chemical Reagent Co., Ltd.). The working solution $(0.3 \%, \mathrm{w} / \mathrm{v})$ was produced by diluting the stock solution with double distilled water.

Diet preparations and feeding systems. To prepare the HCD, regular zebrafish diet (3\% crude fat; SLRC Laboratory Animal Co., Ltd., Shanghai, China) was ground into powder and soaked in chloroform containing cholesterol (SLRC Laboratory Animal Co., Ltd.). Following evaporation of the chloroform, the HCD was ready (4\% cholesterol, w/w).

Chicken's egg yolk powder (Shanghai Yuanye Biotechnology Co., Ltd., Shanghai, China) was used for the HFD and 7-dpf zebrafish were also incubated in a water system containing $0.1 \%(\mathrm{~g} / \mathrm{ml})$ egg yolk for $48 \mathrm{~h}$. The breeding water was replaced with freshly prepared $0.1 \%(\mathrm{~g} / \mathrm{ml})$ egg yolk solution every $4 \mathrm{~h}$. Following $48 \mathrm{~h}$ of incubation, the zebrafish were washed in breeding water and subsequently stained with Oil Red O.

CholEsteryl BODIPY 542/563 C11 was dissolved in chloroform, in which regular zebrafish diet or HCD was soaked. Following complete evaporation of the chloroform, a diet containing 1/100,000 (w/w) fluorescent probe was produced.

Oil Red O staining. Oil Red O staining was performed according to the protocol described in a study by Schlombs et al (15). Briefly, following anesthesia with MS-222 (200 ppm), zebrafish were fixed in 4\% paraformaldehyde for $12 \mathrm{~h}$ at $4^{\circ} \mathrm{C}$ and washed three times in $1 \mathrm{X}$ phosphate-buffered saline. Fish were subsequently preincubated in $60 \%$ isopropanol for $30 \mathrm{~min}$ and dyed with fresh $0.3 \%$ Oil Red O for $3 \mathrm{~h}$. Samples were ready for microscopic observation following three washes with $60 \%$ isopropanol. A Nikon SMZ1500 (Nikon Corporation, Tokyo, Japan) was used for observations and capturing images.

Administration of CholEsteryl BODIPY 542/563 C11. Wild-type zebrafish ( $3 \mathrm{dpf}$ ) were incubated in breeding water containing $0.1 \mathrm{ppm}$ CholEsteryl BODIPY 542/563 C11 for $24 \mathrm{~h}$ to determine the efficiency of CholEsteryl BODIPY 542/563 C11 intravascular lipid staining. Two experimental methods were subsequently used to administer CholEsteryl BODIPY 542/563 C11 to the zebrafish. In one group, 7-dpf wild-type zebrafish were kept in breeding water containing $0.1 \mathrm{ppm}$ CholEsteryl BODIPY 542/563 C11 and fed with a HCD (twice/day) for $10 \mathrm{~d}$. In the second system, 7-dpf wild-type zebrafish were kept in regular breeding water and fed with a regular diet (control group, $n=30$ ) or a HCD (HCD group, $\mathrm{n}=30$ ) supplemented with 1/100,000 (w/w) CholEsteryl BODIPY 542/563 C11 for $10 \mathrm{~d}$. A Nikon ECLIPSE TE2000-E microscope (Nikon Corporation, Tokyo, Japan) was used for observations and capturing images. Images were analyzed using NIS-Elements BR 3.1 software (Nikon Corporation, 
A

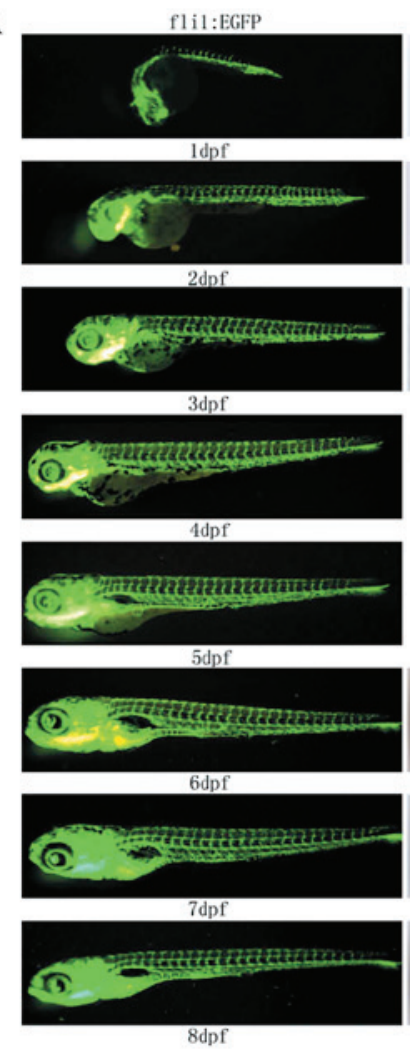

$\mathrm{AB}$
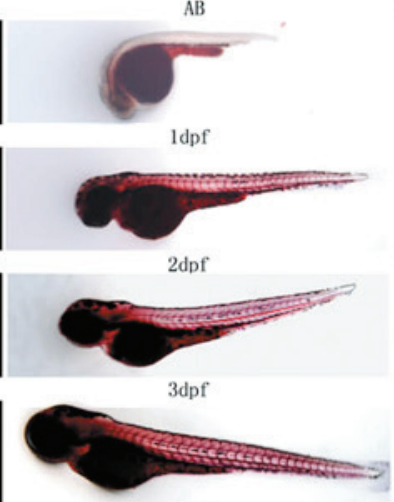

$4 \mathrm{dpf}$

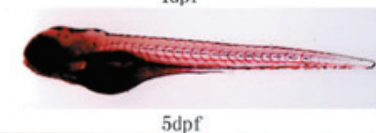

$5 \mathrm{dnf}$

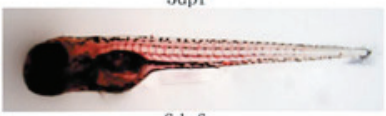

$6 \mathrm{dpf}$

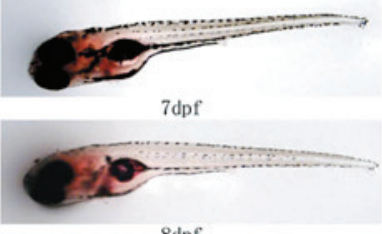

B

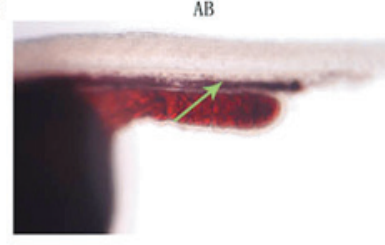

$1 \mathrm{dpf}$

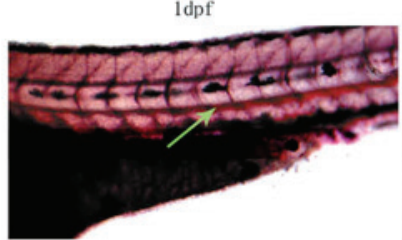

$3 \mathrm{dpf}$

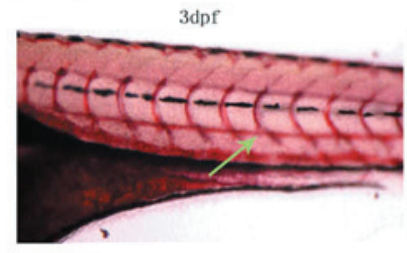

$5 \mathrm{dpf}$

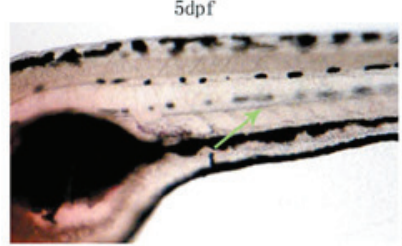

$7 \mathrm{dpf}$

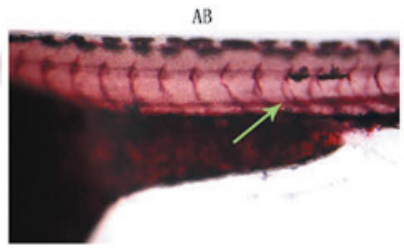

$2 \mathrm{dpf}$

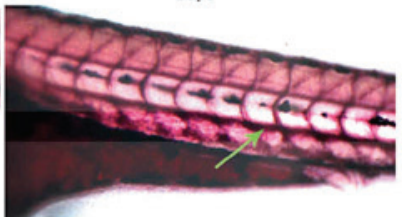

$4 \mathrm{dpf}$

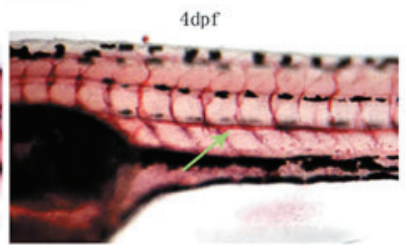

$6 \mathrm{dpf}$

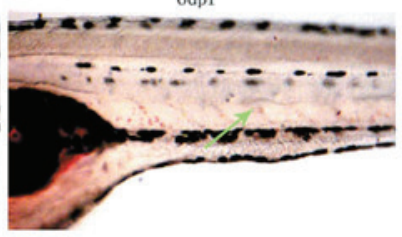

$8 \mathrm{dpf}$

Figure 1. Oil Red O staining of 1-8-dpf fasting zebrafish. (A) fli1:EGFP (first column) and wild-type (AB strain; second column) zebrafish in lateral view with the anterior section to the left (magnification, x30). (B) Enlargement (magnification, x112.5) of AB strain zebrafish at trunk level. Green arrows indicate the dorsal aorta. dpf, days post fertilization.

Tokyo, Japan) to calculate the intra-aortic fluorescence intensity.

Detection of adult zebrafish blood lipids. A total of 100 male zebrafish (age, 13 weeks) were randomly divided into control and HFD groups. In the control group, the zebrafish were fed a regular diet thrice daily for seven weeks. The zebrafish in the HFD group were fed a HCD (twice daily) and a high-fat egg-yolk diet (once daily, in between the HCD feeds) for seven weeks. Any remaining feed was removed $30 \mathrm{~min}$ following each administration.

Zebrafish were deeply anesthetized with MS-222 (200 ppm) and $5 \mu \mathrm{l}$ blood was drawn from the artery of each fish to obtain $1 \mu \mathrm{l}$ serum following centrifugation at $2,500 \mathrm{x} \mathrm{g}$ for $10 \mathrm{~min}$ at $4^{\circ} \mathrm{C}$. A total of 30 zebrafish were randomly selected from each group and the collected serum of five zebrafish was combined as one sample for detection. Total cholesterol and triglycerides in serum were measured using the Siemens Advia 2400 automatic analyzer (Siemens Healthcare, Erlangen, Germany) (11).

Statistical analysis. SPSS 11.5 (SPSS, Inc., Chicago, IL, USA) was used for data analysis. Values are expressed as the mean \pm standard deviation. Student's t-test was used to compare the data between two groups, and data among more than two groups were compared using analysis of variance. $\chi^{2}$ analysis and Fisher's exact test were used accordingly for comparison of rates. $\mathrm{P}<0.05$ was considered to indicate a statistically significant difference between values.

\section{Results}

Oil Red $O$ staining of 1-8-dpf fasting zebrafish. The Oil Red O-stained samples of 1-8-dpf fasting wild-type (AB strain) zebrafish and the 1-8-dpf fli1:EGFP zebrafish were prepared and observed under a fluorescence microscope (Fig. 1A and B). Intravascular lipid levels were reduced with increasing days post-fertilization due to the absorption of the yolk sac. Due to the incomplete blood vessel development, no clear Oil Red O signal for intravascular lipids was detected in 1-dpf zebrafish. Lipid signals were significant in 2-5-dpf zebrafish and comparison to age-matched fli1:EGFP zebrafish indicated that the detected lipids were intravascular. The lipid signal became markedly weaker in 6-dpf zebrafish. Aside from the swim bladder, no lipid signal was detected in the blood vessels of zebrafish older than seven dpf, as the yolk sac had been completely absorbed. These results demonstrated that Oil Red O staining was able to indicate intravascular lipid levels in vivo.

Effects of HFD on the intravascular lipid levels of zebrafish. Fasting 7-dpf zebrafish incubated in breeding water containing $0.1 \%(\mathrm{~g} / \mathrm{ml})$ egg yolk, as a HFD, for $48 \mathrm{~h}$ were stained with Oil Red $\mathrm{O}$ and compared with the fasting zebrafish at 9-dpf (Fig. 2). The Oil Red $\mathrm{O}$ signal for intravascular lipids was not detected in fasting 9-dpf zebrafish (Fig. 2A and B), while the intravascular lipid levels of zebrafish incubated in $0.1 \%$ egg yolk for $48 \mathrm{~h}$ were significantly increased, demonstrated by the greater intensity of Oil Red O staining observed (Fig. 2C and D). 

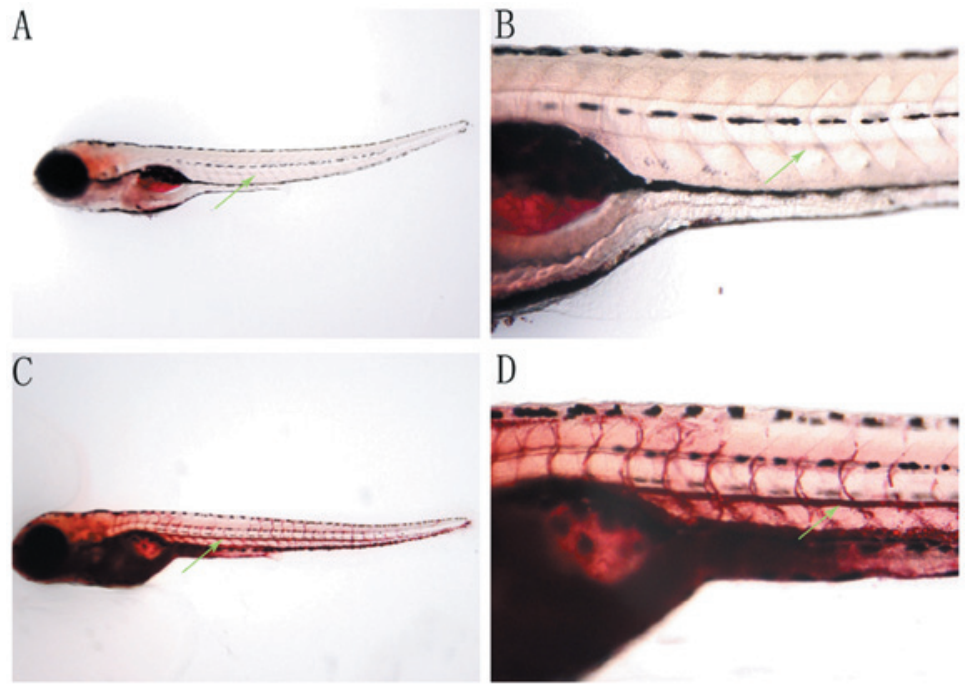

Figure 2. Effect of HFD on zebrafish intravascular lipids via Oil Red O staining. (A) A representative 9 dpf fasting zebrafish in lateral view with the anterior part to the left (magnification, x30). (B) Enlargement of 9-dpf fasting zebrafish at trunk level (magnification, x112.5. (C) A representative 9-dpf zebrafish fed with HFD [7-dpf fasting zebrafish incubated in breeding water containing $0.1 \%(\mathrm{~g} / \mathrm{ml})$ egg yolk for $48 \mathrm{~h}$ ] in lateral view with the anterior part to the left (magnification, x30). (D) Enlargement of the 9-dpf HFD zebrafish at trunk level (magnification, x112.5). Green arrows indicate the dorsal aorta. HFD, high-fat diet; dpf, days post fertilization.
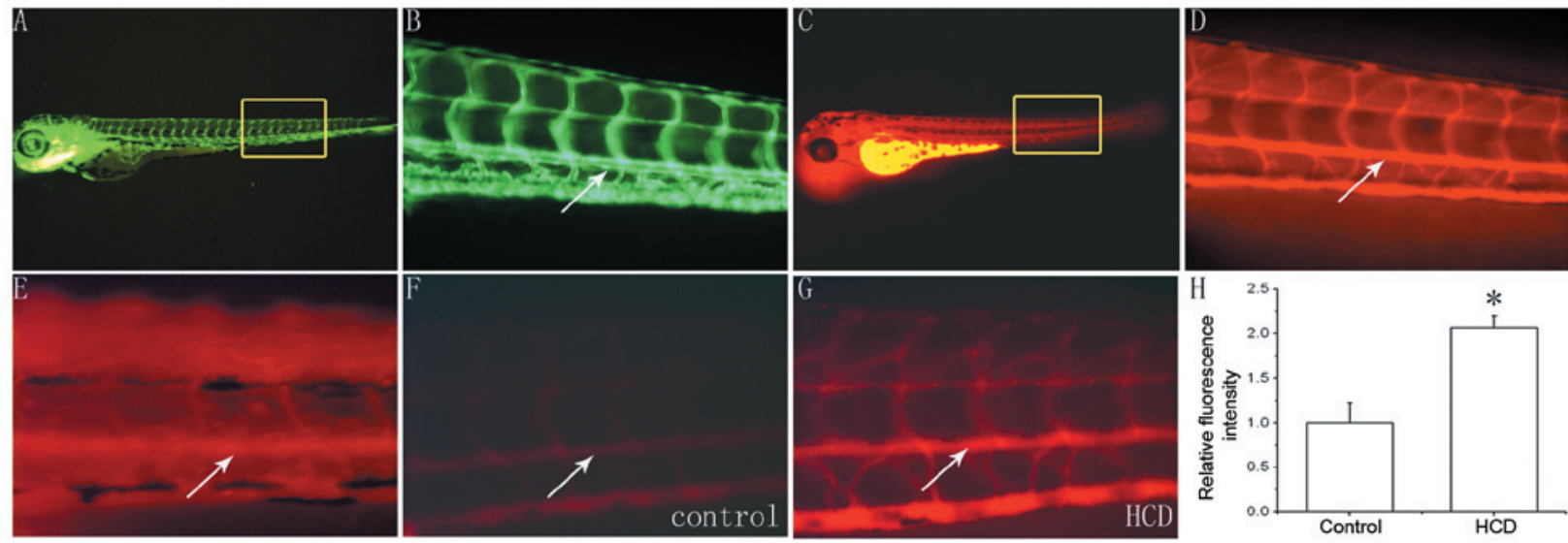

Figure 3. Effects of HCD on the intravascular cholesterol of zebrafish. (A) Four dpf fli1:EGFP zebrafish in lateral view with the anterior section to the left (magnification, x30). (B) Enlargement of the yellow box indicated in A (magnification, x200). (C) Wild-type zebrafish (3 dpf) incubated in breeding water containing $0.1 \mathrm{ppm}$ CholEsteryl BODIPY $542 / 563 \mathrm{C} 11$ for $24 \mathrm{~h}$ in lateral view with the anterior to the left (magnification, $\mathrm{x} 30$ ). (D) Enlargement of the yellow box indicated in C (magnification, x200). (E) Wild-type zebrafish (7 dpf) incubated in breeding water containing 0.1 ppm CholEsteryl BODIPY 542/563 C11 and fed with HCD (twice/day) for $10 \mathrm{~d}$ (magnification, x200). (F) Wild-type zebrafish (7 dpf) incubated in regular breeding water and fed a regular diet containing CholEsteryl BODIPY 542/563 C11 for $10 \mathrm{~d}$ (control; magnification, x200) or (G) HCD containing CholEsteryl BODIPY 542/563 C11 for $10 \mathrm{~d}$ (n=28; magnification, x200). (H) Variation in intra-aortic fluorescence intensity between zebrafish fed a HCD and zebrafish fed a regular diet. "P<0.05 vs.control group. Values are expressed as the mean \pm standard deviation $(n=28)$. White arrows indicate the dorsal aorta of zebrafish. HCD, high cholesterol diet; dpf, days post fertilization.

Staining for intravascular cholesterol of embryonic zebrafish. The 3-dpf wild-type zebrafish incubated in breeding water containing $0.1 \mathrm{ppm}$ CholEsteryl BODIPY 542/563 C11 for $24 \mathrm{~h}$ were observed under a fluorescence microscope. The distribution of red fluorescence observed in these wild-type zebrafish was analogous to that of the blood vessel distribution observed in 4-dpf fli1:EGFP zebrafish (Fig. 3A-D). Therefore, zebrafish intravascular cholesterol was able to be stained by CholEsteryl BODIPY 542/563 C11.

Effects of HCD on zebrafish intravascular cholesterol. Wild-type zebrafish (7-dpf) were incubated in breeding water containing 0.1 ppm CholEsteryl BODIPY 542/563 C11 and fed with the HCD (twice/day) for $10 \mathrm{~d}$. However, it was difficult to accurately calculate intravascular fluorescence intensity as not only the blood vessels, but additionally other areas of the zebrafish expressed red fluorescence (Fig. 3E).

Amongst the 7-dpf wild-type zebrafish kept in regular breeding water and fed a regular diet ( $\mathrm{n}=30$, control group) or the HCD ( $\mathrm{n}=30$, HCD group) containing CholEsteryl BODIPY 542/563 C11 for $10 \mathrm{~d}$, two zebrafish died in each group and there was no significant difference in mortality between the two groups $(\mathrm{P}=1.000)$. The remaining zebrafish were used for observation of intravascular fluorescence intensity by fluorescence microscopy. The intravascular fluorescence intensity of zebrafish in the HCD group was markedly higher than that of 

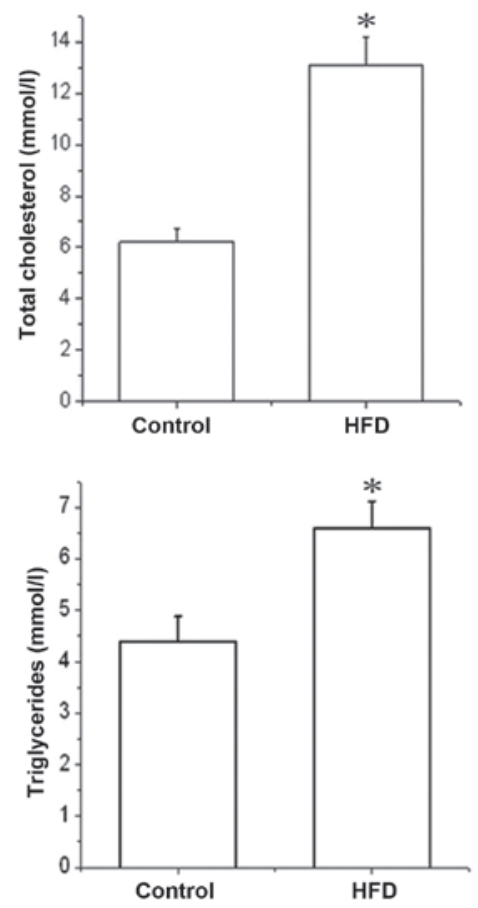

Figure 4. Variation in the serum total cholesterol and triglycerides between adult zebrafish fed with regular diet (control) and HFD for seven weeks. ${ }^{*} \mathrm{P}<0.05$, compared with the control group. HFD, high-fat diet. Values are presented as the mean \pm standard deviation.

those in the control group (Fig. 3F and G). Furthermore, there was a significant difference between the intra-aortic fluorescence intensity of the HCD group and that of the control group $(2.06 \pm 0.14$ vs. $1.00 \pm 0.23, \mathrm{P}<0.05$; Fig. $3 \mathrm{H})$.

Effects of HFD on serum total cholesterol and triglycerides in zebrafish. All zebrafish in the control and HFD groups survived. In each group, 30 zebrafish were randomly selected for detection of the serum total cholesterol and triglycerides (Fig. 4). The serum total cholesterol of zebrafish in the HFD group was significantly higher than that in the control group $(13.1 \pm 1.10$ vs. $6.20 \pm 0.50 \mathrm{mmol}, \mathrm{P}<0.05)$. Furthermore, serum triglyceride levels were also markedly increased in the HFD group compared to those of the control group (6.60 \pm 0.52 vs. $4.38 \pm 0.50 \mathrm{mmol} ; \mathrm{P}<0.05)$.

\section{Discussion}

At present, organism-based models are widely used in small-chemical compound discovery and such animal-based screening models make compound-screening feasible prior to the elucidation of detailed mechanisms of the signaling pathways involved (16-18). For an animal-based model, the animal should be small, low-cost, compatible with simple culture conditions and suitable for high-throughput screening (19). Zebrafish meet these criteria, are able to generate large clutches of eggs and quickly develop most organs, including the blood vessels. Furthermore, numerous gastrointestinal organs, including the intestines, liver, gallbladder, exocrine and endocrine pancreas, as well as the specialized cell types associated with lipid absorption and processing in larval zebrafish were found to be analogous with those of humans $(13,20,21)$.
Therefore, the zebrafish was selected as an experimental model to establish the methods for investigating intravascular lipid levels in the present study.

Convenient and effective methods for intravascular lipid-detection in zebrafish are essential for antihyperlipidemic drug screening. Oil Red O is a low-cost, reproducible and neutral fat-staining agent and is useful in high-throughput research (22). Clifton et al (23) analyzed the effects of certain novel compounds on lipid metabolism in the digestive tract of zebrafish using Oil Red O staining. However, there were few studies investigating the intravascular lipid metabolism of zebrafish using Oil Red O. In the present study, Oil Red O-stained samples of 1-8-dpf zebrafish were prepared and the staining results demonstrated that Oil Red O was able to effectively indicate intravascular lipids in vivo. Fluorescent probes are also useful for dynamic monitoring of zebrafish lipids in vivo (11). Two methods for incorporating the fluorescent probe CholEsteryl BODIPY 542/563 C11 were established. In one group, CholEsteryl BODIPY 542/563 C11 was added into the zebrafish breeding system and in the other, CholEsteryl BODIPY 542/563 C11 was incorporated into the fish diet. When the 3-dpf zebrafish were incubated in breeding water containing 0.1 ppm CholEsteryl BODIPY 542/563 C11 for $24 \mathrm{~h}$, the intravascular cholesterol was stained by CholEsteryl BODIPY 542/563 C11. A previous study has indicated that zebrafish older than 7-dpf must be fed with the HCD for $\geq 10 \mathrm{~d}$ in order to achieve a marked increase in the intravascular cholesterol (11). However, this increased incubation time in breeding water containing CholEsteryl BODIPY 542/563 C11 resulted in not only blood vessels but also other areas of the zebrafish emitting strong red fluorescence, which made accurate quantification of intravascular fluorescence intensity difficult. In the second system, where CholEsteryl BODIPY 542/563 C11 was incorporated into the fish diet, the fluorescence was concentrated in the blood vessels.

The fli1:EGFP transgenic zebrafish expressed an endothelial-specific EGFP that enabled detailed visualization of blood vessels in vivo (24). Therefore, fli1:EGFP zebrafish, which exhibited the distribution of blood vessels, were used for comparison, to ensure that the substances stained with Oil Red O or labeled by CholEsteryl BODIPY 542/563 C11 were intravascular lipids. Zebrafish are optically transparent until 30-dpf, which enables temporal observations of fluorescent probes and dyes in the live animal. Oil Red O staining and fluorescence labeling methods were able to indirectly indicate the relative amount of intravascular lipids, whereas the blood of adult zebrafish could be used to accurately quantify blood lipids. Therefore, blood was collected from male zebrafish aged 13 weeks, and the serum total cholesterol and triglycerides were analyzed in order to validate the accuracy of Oil Red O staining and fluorescence labeling. The results supported those indicated by Oil Red O and CholEsteryl BODIPY 542/563 C11 staining. The use of adult zebrafish for drug screening is unsuitable due to the fact that such experiments using adult zebrafish can be time-consuming and expensive (25).

Since zebrafish eggs and embryos are highly permeable, compounds are able to be added directly to the breeding water and the accessibility of compounds to the embryos is therefore high. Moreover, the use of zebrafish would be convenient for high-throughput screening, as the eggs and larvae are small 
enough to be screened in 96-well plates. Conventionally, chemical libraries are stored as stock solutions in DMSO. Previous studies have demonstrated that zebrafish embryos are tolerant to a range of DMSO concentrations and that $1 \%$ DMSO is compatible with normal development, indicating that compounds are able to be screened at a wide range of concentrations $(26,27)$. For lipid detection and imaging, in vivo fluorescent signals may be detected by automatic fluorescence microscopy in high-throughput screening (28).

Future studies may focus on the development of lower vertebrate models of human diseases, for example mutant zebrafish carrying mutations in the lipid metabolism pathway (29-31). The effects of expressional changes in genes associated with regulation of intravascular lipids induced by Morpholinoor messenger RNA injections may be investigated using Oil Red O staining or breeding water containing CholEsteryl BODIPY 542/563 C11. Alternatively, Oil Red O may be used to analyze drug efficacy in 7-dpf zebrafish fed with an HFD following $48 \mathrm{~h}$ of incubation in breeding water containing drugs. Drug efficacy may also be studied in 7-dpf zebrafish fed with an HFD labeled with CholEsteryl BODIPY 542/563 C11 using a fluorescence microscope following incubation in breeding water containing drugs for $10 \mathrm{~d}$. The hypolipidemic effects of screened drugs may be validated in adult zebrafish kept in a medicinal bath and fed an HFD.

In conclusion, the methods of Oil Red O staining and CholEsteryl BODIPY 542/563 C11 administered via breeding water or diet were optimized for zebrafish intravascular lipid metabolism research. These methods may be applied to novel lipid-regulating drug screening and investigations into genes associated with lipid regulation.

\section{Acknowledgements}

The present study was supported by the National Natural Science Foundation of China (grant nos. 30971436, 31201010, and 81270376), and the Foundation of Shanghai Ninth People's Hospital (grant no. 2013A02).

\section{References}

1. Joffe BI, Panz VR and Raal FJ: From lipodystrophy syndromes to diabetes mellitus (Review). Lancet 357: 1379-1381, 2001.

2. McNeely MJ, Edwards KL, Marcovina SM, Brunzell JD, Motulsky AG and Austin MA: Lipoprotein and apolipoprotein abnormalities in familial combined hyperlipidemia: a 20-year prospective study. Atherosclerosis 159: 471-481, 2001

3. Watanabe S, Yaginuma R, Ikejima K and Miyazaki A: Liver diseases and metabolic syndrome (Review). J Gastroenterol 43 : 509-518, 2008

4. Rocke J, Lees J, Packham I and Chico T: The zebrafish as a novel tool for cardiovascular drug discovery (Review). Recent Pat Cardiovasc Drug Discov 4: 1-5, 2009.

5. Hölttä-Vuori M1, Salo VT, Nyberg L, Brackmann C, Enejder A, Panula P and Ikonen E: Zebrafish: gaining popularity in lipid research (Review). Biochem J 429: 235-242, 2010.

6. Schirmer H, Pereira TC, Rico EP, Rosemberg DB, Bonan CD, Bogo MR and Souto AA: Modulatory effect of resveratrol on SIRT1, SIRT3, SIRT4, PGC1 $\alpha$ and NAMPT gene expression profiles in wild-type adult zebrafish liver. Mol Biol Rep 39: 3281-3289, 2012.

7. Zhang Y, Hu G, Li S, Li ZH, Lam CO, Hong SJ, Kwan YW, Chan SW, Leung GP and Lee SM: Pro-angiogenic activity of astragaloside IV in HUVECs in vitro and zebrafish in vivo. Mol Med Rep 5: 805-811, 2012.

8. Carten JD and Farber SA: A new model system swims into focus: using the zebrafish to visualize intestinal lipid metabolism in vivo. Clin Lipidol 4: 501-515, 2009.
9. Hama K, Provost E, Baranowski TC, Rubinstein AL, Anderson JL, Leach SD and Farber SA: In vivo imaging of zebrafish digestive organ function using multiple quenched fluorescent reporters. Am J Physiol Gastrointest Liver Physiol 296: G445-G453, 2009.

10. Farber SA, Pack M, Ho SY, Johnson ID, Wagner DS, Dosch R, Mullins MC, Hendrickson HS, Hendrickson EK and Halpern ME: Genetic analysis of digestive physiology using fluorescent phospholipid reporters. Science 292: 1385-1388, 2001.

11. Stoletov K, Fang L, Choi SH, Hartvigsen K, Hansen LF, Hall C, Pattison J, Juliano J, Miller ER, Almazan F, et al: Vascular lipid accumulation, lipoprotein oxidation, and macrophage lipid uptake in hypercholesterolemic zebrafish. Cir Res 104: 952-960, 2009.

12. Kang OH, Kim SB, Seo YS, Joung DK, Mun SH, Choi JG, Lee YM, Kang DG, Lee HS and Kwon DY: Curcumin decreases oleic acid-induced lipid accumulation via AMPK phosphorylation in hepatocarcinoma cells. Eur Rev Med Pharmacol Sci 17: 2578-2586, 2013

13. Schlegel A and Stainier DY: Microsomal triglyceride transfer protein is required for yolk lipid utilization and absorption of dietary lipids in zebrafish larvae. Biochemistry 45: 15179-15187, 2006.

14. Marks DL, Bittman R and Pagano RE: Use of Bodipy-labeled sphingolipid and cholesterol analogs to examine membrane microdomains in cells. Histochem Cell Biol 130: 819-832, 2008.

15. Schlombs K, Wagner T and Scheel J: Site-1 protease is required for cartilage development in zebrafish. Proc Natl Acad Sci USA 100: 14024-14029, 2003.

16. Murphey RD, Stern HM, Straub CT and Zon LI: A chemical genetic screen for cell cycle inhibitors in zebrafish embryos. Chem Biol Drug Des 68: 213-219, 2006.

17. Gosai SJ, Kwak JH, Luke CJ, Long OS, King DE, Kovatch KJ, Johnston PA, Shun TY, Lazo JS, Perlmutter DH, et al: Automated high-content live animal drug screening using $C$. elegans expressing the aggregation prone serpin $\alpha 1$-antitrypsin Z. PLoS One 5: e15460, 2010.

18. Wheeler GN and Brändli AW: Simple vertebrate models for chemical genetics and drug discovery screens: lessons from zebrafish and Xenopus. Dev Dyn 238: 1287-1308, 2009.

19. Peterson RT, Link BA, Dowling JE and Schreiber SL: Small molecule developmental screens reveal the logic and timing of vertebrate development. Proc Natl Acad Sci USA 97: 12965-12969, 2000.

20. Lieschke GJ and Currie PD: Animal models of human disease: zebrafish swim into view (Review). Nat Rev Genet 8: 353-367, 2007.

21. Wallace KN, Akhter S, Smith EM, Lorent K and Pack M: Intestinal growth and differentiation in zebrafish. Mech Dev 122: $157-173,2005$.

22. Mehlem A, Hagberg CE, Muhl L, Eriksson U and Falkevall A: Imaging of neutral lipids by oil red $\mathrm{O}$ for analyzing the metabolic status in health and disease. Nat Protoc 8: 1149-1154, 2013.

23. Clifton JD, Lucumi E, Myers MC, Napper A, Hama K, Farber SA, Smith AB III, Huryn DM, Diamond SL and Pack M: Identification of novel inhibitors of dietary lipid absorption using zebrafish. PLoS One 5: e12386, 2010.

24. Lawson ND and Weinstein BM: In vivo imaging of embryonic vascular development using transgenic zebrafish. Dev Biol 248: 307-318, 2002.

25. Terriente $\mathbf{J}$ and Pujades $\mathrm{C}$ : Use of zebrafish embryos for small molecule screening related to cancer. Dev Dyn 242: 97-107, 2013.

26. Brändli AW: Prospects for the Xenopus embryo model in therapeutics technologies. CHIMIA 58: 694-702, 2004.

27. Chan J and Serluca FC: Chemical approaches to angiogenesis. Methods Cell Biol 76: 475-487, 2004.

28. Starkuviene V and Pepperkok R: The potential of highcontent high-throughput microscopy in drug discovery. Br J Pharmacol 152: 62-71, 2007.

29. Hong CC, Peterson QP, Hong J-Y and Peterson RT: Artery/vein specification is governed by opposing phosphatidylinositol-3 kinase and MAP kinase/ERK signaling. Curr Biol 16: 1366-1372, 2006.

30. Peterson RT and Fishman MC: Discovery and use of small molecules for probing biological processes in zebrafish. Methods Cell Biol 76: 569-591, 2004.

31. Stern HM, Murphey RD, Shepard JL, Amatruda JF, Straub CT, Pfaff KL, Weber G, Tallarico JA, King RW and Zon LI: Small molecules that delay $S$ phase suppress a zebrafish bmyb mutant. Nat Chem Biol 1: 366-370, 2005. 